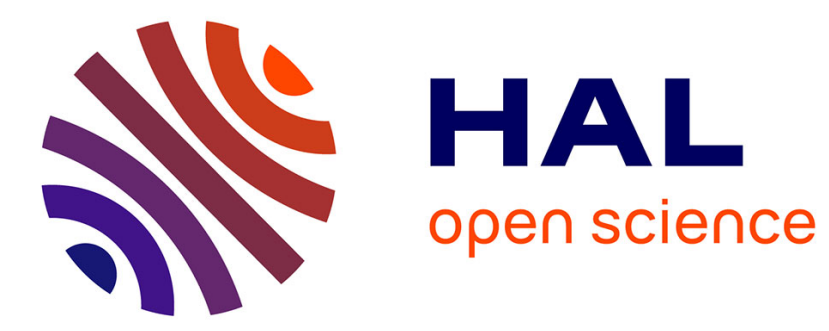

\title{
Comparison of gadolinium nanoparticles and molecular contrast agents for radiation therapy-enhancement
}

\author{
R. Delorme, Florence Taupin, Mélanie Flaender, Jean-Luc Ravanat, \\ Christophe Champion, Mathieu Agelou, Hélène Elleaume
}

\section{- To cite this version:}

R. Delorme, Florence Taupin, Mélanie Flaender, Jean-Luc Ravanat, Christophe Champion, et al. Comparison of gadolinium nanoparticles and molecular contrast agents for radiation therapyenhancement. Medical Physics, 2017, 44 (11), pp.5949-5960. 10.1002/mp.12570 . hal-01690606

\section{HAL Id: hal-01690606 https://hal.science/hal-01690606}

Submitted on 1 Oct 2019

HAL is a multi-disciplinary open access archive for the deposit and dissemination of scientific research documents, whether they are published or not. The documents may come from teaching and research institutions in France or abroad, or from public or private research centers.
L'archive ouverte pluridisciplinaire HAL, est destinée au dépôt et à la diffusion de documents scientifiques de niveau recherche, publiés ou non, émanant des établissements d'enseignement et de recherche français ou étrangers, des laboratoires publics ou privés. 


\section{Comparison of Gadolinium Nanoparticles and Molecular Contrast Agents for Radiation Therapy-Enhancement}

\section{DELORME Rachel ${ }^{1,7}$, TAUPIN Florence ${ }^{2,3,6,{ }^{*},}$ FLAENDER Mélanie ${ }^{2,3,6,{ }^{*},}$ RAVANAT Jean-Luc ${ }^{5,6}$,} CHAMPION Christophe ${ }^{4}$, AGELOU Mathieu ${ }^{1}$, ELLEAUME Hélène ${ }^{2,3}$

${ }^{1}$ CEA, LIST, F-91191 Gif-sur-Yvette, France

${ }^{2}$ Université Grenoble Alpes, EA-7442 Rayonnement Synchrotron et Recherche Médicale, F-38058 Grenoble cedex 9, France

${ }^{3}$ European Synchrotron Radiation Facility, F-38000, Grenoble, France

${ }^{4}$ Centre d'Études Nucléaires de Bordeaux Gradignan, CENBG, CNRS/IN2P3, Université de Bordeaux, France

${ }^{5}$ Laboratoire "Lésions des Acides Nucléiques", Univ. Grenoble Alpes, INAC-SCIB, F-38000 Grenoble, France

${ }^{6} \mathrm{CEA}$, INAC-SCIB, F-38000 Grenoble, France

${ }^{7}$ IMNC Laboratory, UMR 8165-CNRS/IN2P3, Paris-Saclay university, 91405 Orsay, France

* Authors equally contributed to this study

Keywords: gadolinium, nanoparticles, contrast agents, radiation therapy, dose-enhancement, Monte Carlo simulations

Corresponding author: delorme@imnc.in2p3.fr 
Abstract

Purpose: Nanoparticles appear as a novel tool to enhance the effectiveness of radiotherapy in cancer treatments. Many parameters influence their efficacy, such as their size, concentration, composition, their cellular localization, as well as the photon source energy. The current Monte Carlo study aims at comparing the dose-enhancement in presence of gadolinium (Gd), either as isolated atoms or atoms clustered in nanoparticles (NPs), by investigating the role played by these physical parameters at the cellular and the nanometer scale. In parallel, in vitro assays were performed in presence of either the gadolinium contrast agent (GdCA) Magnevist ${ }^{\circledR}$ or ultrasmall gadolinium NPs (GdNPs, $3 \mathrm{~nm}$ ) for comparison with the simulations.

Methods: PENELOPE Monte Carlo Code was used for in silico dose calculations. Monochromatic photon beams were used to calculate dose-enhancements in different cell compartments and low-energy secondary electron spectra dependence with energy. Particular attention has been placed on the interplay between the X-ray beam energy, the Gd localization and its distance from cellular targets. Clonogenic assays were used to quantify F98 rat glioma cell survival after irradiation in the presence of GdNPs or GdCA, using monochromatic X-rays with energies in the $30 \mathrm{keV}-80 \mathrm{keV}$ range from a synchrotron and $1.25 \mathrm{MeV}$ gamma photons from a cobalt-60 source. The simulations that correspond to the experimental conditions were compared with the experimental results.

Results: In silico, a highly heterogeneous and clustered Gd-atom distribution, a massive production of low energy electrons around GdNPs and an optimal X-ray beam energy, above the Gd K-edge, were key factors found to increase microscopic doses, which could potentially induce cell death. The different Gd localizations studied all resulted in a lower dose enhancement for the nucleus component than for cytoplasm or membrane compartments, with a maximum doseenhancement factor (DEF) found at $65 \mathrm{keV}$ and $58 \mathrm{keV}$, respectively. In vitro, radiosensitization was observed with GdNPs incubated $5 \mathrm{~h}$ with the cells $(2.1 \mathrm{mg} \mathrm{Gd} / \mathrm{mL})$ at all energies. Experimental 
57 DEFs were found to be greater than computational DEFs but follow a similar trend with irradiation energy. However, an important radiosensitivity was observed experimentally with GdNPs at high

59 energy (1.25 MeV), whereas no effect was expected from modeling. This effect was correlated with GdNPs incubation time. In vitro, GdCA provided no dose-enhancement at $1.25 \mathrm{MeV}$ energies,

61 in agreement with computed data.

62 Conclusions: These results provide a foundation on which to base optimizations of the physical 63 parameters in Gd radiation-enhanced therapy. Strong evidence was provided that GdCA or GdNPs could both be used for radiation dose-enhancement therapy. Their in vivo biological distribution,

65 in the tumor volume and at the cellular scale, will be the key factor for providing large dose66 enhancements and determine their therapeutic efficacy. 


\section{Introduction}

An innovative therapeutic approach using heavy elements (i.e. high atomic number) in conjunction with low-energy radiation of the order of tens to hundreds of keV, seems to offer a promising approach for the treatment of resistant cancers. Indeed, loading tumors with heavy elements results in a differential effect between the tumor and the surrounding unloaded healthy tissue due to the large increase in low energy X-ray absorption in these elements. This effect is defined as "radiation dose-enhancement". Many studies have been published, with a particular emphasis in the last decade, on the use of nanoparticles (NP) replacing contrast agents (CA) as radiation dose-enhancers. Numerous in vivo and in vitro experiments have shown a significant increased efficacy in the presence of gold $\left(Z_{A u}=79\right)^{1-5}$ or gadolinium $\left(Z_{G d}=64\right)^{6-8} \mathrm{NPs}$, but the underlying mechanisms leading to increased cell kill are still unclear. This efficacy can be attributed in part to physical aspects, such as the macro- and nano-scale radiation dose-enhancement, but also to additional chemical and biological mechanisms, here defined as radiosensitization, such as high-density reactive oxygen species (ROS) generation, cell cycle effects ${ }^{2}$, DNA reparation impairment and cytoplasmic events due to damage to the lysosomal system ${ }^{9}$ or to mitochondria ${ }^{10}$. Numerical investigations showed the importance of considering the heterogeneous distribution of high- $Z$ atoms at the micrometer scale ${ }^{11,12}$ as well as the influence of the NP structure at the nanometer scale ${ }^{13-21}$ to improve the description of gold NP (AuNP) radiation interactions. To better understand and optimize the dose-enhancement and toxicity effects of high-Z NPs, it is of great importance to study in detail the influence of beam energy. The main advantage of using low energy photon beams is the large cross sections of photoelectric (PE) interactions on the $\mathrm{K}$ and $\mathrm{L}$ shells of heavy materials, whereas high-energy beams predominantly induce Compton interactions. PE interaction is followed by an atomic reorganization that leads to the emission of a large number of Low-Energy Electrons (LEE: photoelectrons, Auger and Coster-Kroenig electrons) whose relative biological efficiency has been noted in the past and place LEE as a major responsible for the effectiveness of NP (see, e.g. 
the review of Nikjoo et $a .^{22}$ ). The prior work of McMahon et al. ${ }^{19,23}$ has demonstrated very high dose heterogeneities in the near vicinity (a few hundred nanometers) of an AuNP and attributed it to the large number of LEE produced. Assuming the AuNP location in the cell nuclei, their biological efficiency have been well correlated to in vitro results by combining these nanometric doses and the Local Effect Model ${ }^{17,19}$. However, the NPs currently used in in vivo and in clonogenic assays are often located outside the cell nucleus $7,9,24,25$. The question of other cellular targets then arises with the need of modelling realistic distributions of NPs within a cell to take into account these distances. The impact on dose distribution of Au-clusters located in the cytoplasm ${ }^{15}$ or AuNPs located around mitochondria ${ }^{18,26}$ have been investigated with a compartmentalized cell model. Although most of the literature is focused on AuNP dose-enhancement, recent experimental studies have promoted the use of gadolinium nanoparticles (GdNP), by demonstrating both cellular and in vivo increased efficacy ${ }^{7-9,24,27}$. Some of GdNPs (AGulX ${ }^{\circledR}$ NPs) have proved to be of interest for in vivo imaging and for image-guided radiation therapy, because of their paramagnetic properties used in MRI techniques ${ }^{6,28}$. Verry et al. ${ }^{29}$ in a recent study announced their use in an ongoing Phase I clinical trial (Grenoble University hospital, France). It is therefore especially important to better characterize GdNPs behavior under irradiation. In a previous study ${ }^{7}$, we reported large sensitizationenhancement ratio (SER) measured by clonogenic assays, when F98 cells (rodent glioma cells) were irradiated after incubation with ultra-small GdNPs, both in the kilo-voltage energy range (31-80 keV) and at high energy (1.25 MeV). In the same study, we evaluated the radio-sensitizing effect of gadolinium contrast agent (GdCA) using various concentrations $(2.1,5$ and $10 \mathrm{mg} / \mathrm{mL}$ ) of $\mathrm{Gd}$ and we observed radiosensitization only in the kilo-voltage energy range. For cells irradiated in presence of GdCA, the SER profile versus $x$-ray energy was in good agreement with the macroscopic doseenhancement calculated by Monte Carlo simulations. These simulations however could not describe the SER profile versus x-ray energy obtained when the cells were irradiated in presence of GdNPs ${ }^{7}$. 
In the present study, complementary clonogenic experiments were performed with GdNPs

117 and GdCA. The F98 cells were incubated during 5h with GdNPs and then rinsed before irradiation,

118 for removing the contribution of GdNPs in the culture medium. This treatment condition differs 119 from the previous study, where the GdNPs remained in the culture medium during the irradiation.

120 Cell survival was measured after irradiation at various monochromatic X-ray beam energies (from $12131 \mathrm{keV}$ to $80 \mathrm{keV}$ ) at the European Synchrotron Radiation Facility (ESRF, Grenoble, France) and using 122 a Cobalt-60 source (mean energy $1.25 \mathrm{MeV}$ ). Radiation produced by synchrotron source provides a 123 unique tool for evaluating the mechanisms by which radiosensitization and radiation dose124 enhancement occur since it is possible to tune monochromatic X-ray beams over a broad energy 125 range. The dose-enhancement factor (DEF) was defined as the ratio of the dose in presence of Gd 126 relative to the dose without $\mathrm{Gd}$. In the Monte Carlo simulations, the role of the Gd micro-distribution 127 (homogeneously distributed Gd-atoms or clustered Gd-atoms in nanospheres), as well as that of the 128 primary beam energy, were investigated at the sub-cellular scale, in terms of secondary particles 129 generated from Gd ionization and DEF within different cell compartments (membrane, cytoplasm 130 and nucleus). The homogeneously distributed Gd-atoms configuration was used to represent GdCA 131 while clustered Gd-atoms in nanospheres were used to represent isolated GdNP or accumulation of 132 GdNP in Lysosomes. At the nanometer scale, the dose distribution around a single GdNP varying in 133 size was studied for various primary beam energies. The objectives of this study were, firstly, to 134 evaluate by Monte Carlo simulations the dose-enhancement produced by Gd at the sub-cellular and 135 nanometer scale and secondly, to compare selected simulations with the corresponding 136 experimental data.

\section{I.1. Experimental parameters}


Experimental dose-enhancements induced by ultra-small GdNPs and the GdCA Magnevist ${ }^{\circledR}$ were evaluated in vitro by clonogenic assays performed at different energies.

Gd compounds: The GdNPs were provided by the laboratory of O. Tillement (Institut Lumière Matière, univ Lyon, 69622 Villeurbanne cedex, France). These NPs were made of gadolinium chelates (diethylenetriaminepentaacetic acid (DTPA)) covalently grafted to a polysiloxane inorganic matrix. Theses nanoparticles had a mean hydrodynamic diameter of $3 \mathrm{~nm} \pm 1.0 \mathrm{~nm}$ (full description of their synthesis is given in Di Corato et al. $^{30}$ ). The GdCA Magnevist ${ }^{\circledR}$, is a complex of Gd with the same chelating agent, DTPA.

Cell irradiations: F98 rat glioma cells (American Type Culture Collection, Manassas, VA (ATCC, \# CRL2397)) were irradiated in suspension in a volume of $500 \mu \mathrm{L}$ of DMEM in $1.5 \mathrm{~mL}$ Eppendorf tubes. Low energy irradiations ( 31 to $80 \mathrm{keV}$ ) were performed at the ESRF medical beamline (ESRFbiomedical ID17 beamline $-\Delta \mathrm{E} / \mathrm{E} \approx 0.1 \%$ ). The dosimetry was performed using an ionization chamber (PTW Semiflex ion chamber $31010-0.125 \mathrm{~cm}^{3}$ ) placed into an Eppendorf tube. The ionization chamber was scanned vertically through the beam ( $2 \mathrm{~mm}$ in height and $50 \mathrm{~mm}$ in width) at a speed of $2.5 \mathrm{~mm} / \mathrm{s}$ to measure the dose rate. Taking into account, the ring current and the dose rate, the number of scans to deliver $4 \mathrm{~Gy}$ to the cells was calculated. High-energy irradiations were performed at the NUCLEART facility (CEA, Grenoble, France), using a cobalt-60 source, whose gamma emissions are 1.17 and $1.33 \mathrm{MeV}$ (1.25 MeV mean energy). For all conditions, the cells were irradiated at a single dose (D) of 4 Gy evaluated in water.

Cell survival study versus X-ray energy after 5 incubation with GdNPs: in a first experiment, F98 cells were incubated for 5 hours in culture medium (DMEM) containing GdNPs at a concentration of $2.1 \mathrm{mg} \mathrm{Gd} / \mathrm{mL}$ and then rinsed before irradiation with beam energies from $31 \mathrm{keV}$ to $1.25 \mathrm{MeV}$. The GdNPs uptake by the cells was determined by means of inductively coupled plasma-mass 
163

164

spectrometry (ICP-MS). The "control" conditions correspond to cells irradiated at $4 \mathrm{~Gy}$ at each of the above mentioned energies without GdNPs.

Cell survival study at high energy (1.25 MeV): A second experiment was performed to evaluate the influence of incubation time and Gd molecular shape on the cell radiosensitization at high energy. Five treatment conditions were evaluated: (1) control cells (i.e. 4 Gy irradiation alone); (2) cells irradiated in the presence of $2.1 \mathrm{mg} \mathrm{Gd} / \mathrm{mL}$ Magnevist ${ }^{\circledast}(G d C A) ;(3)$ cells irradiated in the presence of $2.1 \mathrm{mg} \mathrm{Gd} / \mathrm{mL}$ of GdNPs (GdNP); (4) cells incubated for 5h with $2.1 \mathrm{mg} \mathrm{Gd} / \mathrm{mL} \mathrm{GdNPs,} \mathrm{rinsed} \mathrm{and}$ irradiated (GdNP-5h-rinsed); and (5) cells incubated for 5h with $2.1 \mathrm{mg} \mathrm{Gd} / \mathrm{mL} \mathrm{GdNPs}$ and irradiated (GdNP-5h).

Clonogenic assay: Three different cells concentrations were seeded in triplicate into Petri dishes (100 mm diameter) containing $8 \mathrm{~mL}$ of complete DMEM, and they were incubated at $37^{\circ} \mathrm{C}$ in an atmosphere containing 95\% air and 5\% CO2 for 11 days. All experiments were repeated three times. Following staining with crystal violet, colonies of greater than 50 cells were enumerated ${ }^{7}$. The surviving fractions (SF) were determined as the ratio of the number of colonies counted divided by the number of cells plated, normalized to non-irradiated controls. The survival fraction ( $\mathrm{SF}_{\text {controls }}$ ) of cells irradiated without Gd versus x-ray dose provided the alpha and beta parameters of the linear quadratic (LQ) model used to fit the survival plots (Eq. 1). These parameters were evaluated at three energies: $33 \mathrm{keV}, 50 \mathrm{keV}$ and $1.25 \mathrm{MeV}$.

$S F_{\text {control }}=\exp \left(-\alpha D-\beta D^{2}\right)$ Equation 1

Sensitization-enhancement ratio and experimental DEF: The Sensitizing Enhancement Ratio versus energy (SER) was defined as the ratio of the SF for control cells to that of cells irradiated with gadolinium either as contrast agent or in the form of GdNP, (SFGd) (Eq. 2).

$\boldsymbol{S E R}=\frac{S F_{\text {control }}}{S F_{G d}}$ Equation 2 
Assuming that the sensitizing enhancement ratio measured in presence of $\mathrm{Gd}$ was uniquely induced by "physical dose-enhancement" (DEF), we could estimate an "experimental dose-enhancement factor" (DEFexp) (Eq. 3 to 5).

$\left.S F_{G d}=\exp \left(-\alpha D \times D E F_{\text {exp }}-\beta\left(D \times D E F_{\text {exp }}\right)^{2}\right)\right) \quad$ Equation 3

$S E R=\exp \left(\alpha D \times\left(D E F_{\text {exp }}-1\right)+\beta D^{2}\left(D E F_{\text {exp }}{ }^{2}-1\right)\right) \quad$ Equation 4

From this expression, one can calculate the $\mathrm{DEF}_{\exp }$ by resolving equation 4 :

$\boldsymbol{D} \boldsymbol{E}_{\boldsymbol{e x p}}=\frac{-\alpha D+\sqrt{(\alpha D)^{2}+4 \beta D^{2} \times\left(\alpha D+\beta D^{2}+l(S E R)\right)}}{2 \beta D^{2}}$ Equation 5

Three energy ranges were defined: low (31 - $40 \mathrm{keV})$, intermediate (50-80 keV) and high (1.25 MeV) energy range. The $\alpha$ and $\beta$ parameters obtained at $33 \mathrm{keV}, 50 \mathrm{keV}$ and $1.25 \mathrm{MeV}$ were used to calculate the SER in these three energy ranges, respectively.

Simulation parameters: Gd distributions were chosen to model the GdCA and GdNPs as homogeneously distributed Gd-atoms or clustered Gd-atoms in nanospheres, respectively. Images taken by confocal microscopy showed that, when incubated $2 \mathrm{~h}$ and $5 \mathrm{~h}$ with F98 cells, the GdNPs agglomerate in clusters around the cell membrane (see Supplemental Material Data). To take into account these clustered conditions and compare the computational DEF with experimental DEF versus photon energy, the GdNPs were modeled as Gd-nanospheres of $50 \mathrm{~nm}$ radius randomly distributed around the cell membrane. This geometry was also used for the experimental comparison at high-energy (GdNP-5h-rinsed). The three other conditions at high energy were modeled using a homogeneous mixture of water and free Gd-atoms in an extracellular medium for the $G d C A$ condition, $50 \mathrm{~nm}$ radius GdNPs randomly distributed in an extracellular medium for the GdNPs condition and also distributed in the cell cytoplasm for the GdNPs-5h condition. The cell geometry is described in Section I.2. As the energy bandwidth of the synchrotron beam on the ID17 beamline is very small $(\Delta E / E=0.1 \%)$, monochromatic photon beams were used in the simulations. 
Additional configurations were considered using Monte Carlo simulations to simulate geometries described in the literature $9,24,25,31$.

\section{I.2. Monte Carlo Simulations at the Sub-Cellular Scale}

A modified and parallelized version of the PENELOPE code was used to take advantage of its variance reduction tool that is well adapted to problems of low probability radiation interactions with nanoparticles ${ }^{32}$. We did not use the default PENELOPE variance reduction technique for "interaction forcing", but a technique developed in the laboratory. For each photon entering the volume of interest, the photon is split into two parts: one that undergoes interaction and one that continues without interacting until the next volume on the trajectory. In this way, the dose is calculated with much lower statistical uncertainty in the volume of interest and, weighted by the adapted probabilities, remain consistent with actual physical cross-sections. The PENELOPE code generates electron and positron histories based on a mixed procedure. The electron transport level of detail is controlled in PENELOPE by specifying the values of several parameters, viz. $C_{1}, C_{2}, W_{C C}$ and $W_{C R}$. The $C_{1}$ and $C_{2}$ parameters are associated with the gathering of elastic scattering processes for electrons and positrons. $W_{C C}$ and $W_{C R}$, represent the cut-off energy loss for, respectively, hard inelastic collisions and hard Bremsstrahlung emission. A detailed description of the algorithms used in PENELOPE can be found in Salvat et al. ${ }^{33}$. The present simulations were done with detailed eventby-event transport setting $C_{1}=C_{2}=0, W_{C C}=50 \mathrm{eV}, W_{C R}=50 \mathrm{eV}$ and using $50 \mathrm{eV}$ as the lowest absorption energy, PENELOPE's low energy threshold.

We used a compartmentalized cell model ${ }^{15,26,34}$, that consists of a single spherical cell of $10 \mu \mathrm{m}$ diameter (the mean diameter of F98 cells was estimated from the fluoroscopy images, see supplementary data file) with a $7.5 \mathrm{~nm}$ thick membrane and a $4 \mu \mathrm{m}$ diameter spherical and centered nucleus. The cell was included in a cube of $15 \mu \mathrm{m}$ side which represents the extracellular medium. All compartments were filled with water by default. The primary X-rays were monochromatic and 
came from a non-divergent square source with $15 \mu \mathrm{m}$ width, placed before the cube. Threedimensional dose-enhancement maps (voxels of $150 \mathrm{~nm}$ ) and mean DEFs in nucleus, cytoplasm and membrane were calculated for various intracellular gadolinium distributions: A) Gd-atoms uniformly distributed in the cytoplasm; B) Gd-atoms agglomerated in $290 \mathrm{~nm}$ diameter spheres simulating lysosomes; C) $50 \mathrm{~nm}$ radius GdNP spheres randomly distributed in the cytoplasm; D) $50 \mathrm{~nm}$ radius GdNP spheres randomly distributed over the cell membrane. The geometries are shown in Figure 1. Configuration $A$ simulates GdCA cytoplasmic internalization, as observed in vitro by De Stasio et al. ${ }^{31}$. Configuration $D$ simulates the experimental study previously described with GdNPs. The $B$ and $C$ configurations correspond to other internalization of nanoparticles in the cytoplasm, for example NPs aggregating in clusters or accumulating in vesicles, as described in the literature ${ }^{9,24,25,35}$. For all conditions, the gadolinium mass was $0.6 \mathrm{pg} /$ cell, to simulate the measured GdNPs cellular uptake. The monochromatic photon energies range from 25 to $1250 \mathrm{keV}$, with a special focus above/below the Gd K-edge (50.24 keV).

\section{A)}

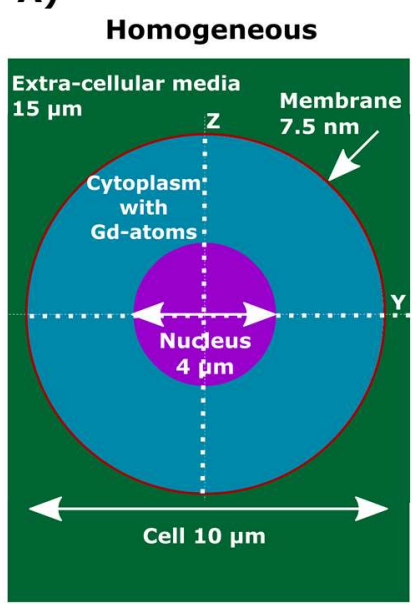

B)

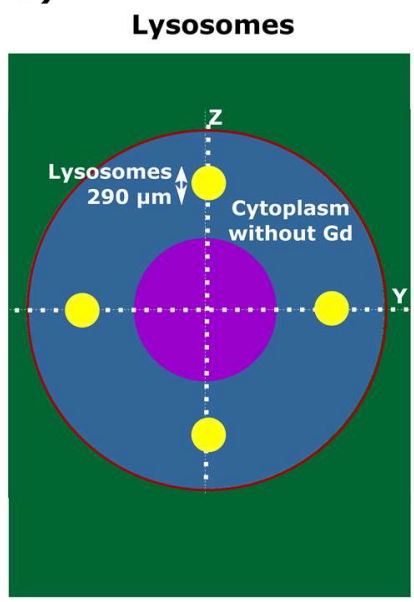

C) GdNP inside cytoplasm

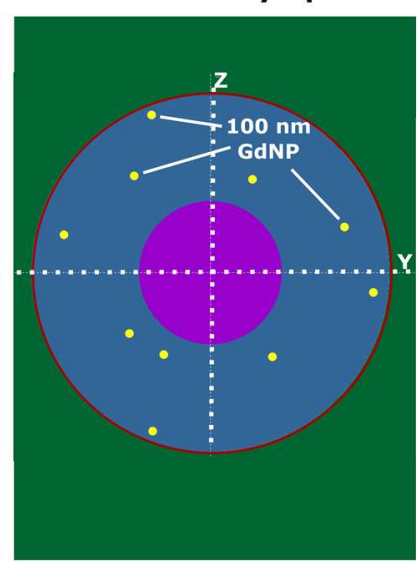

D) GdNP on membrane

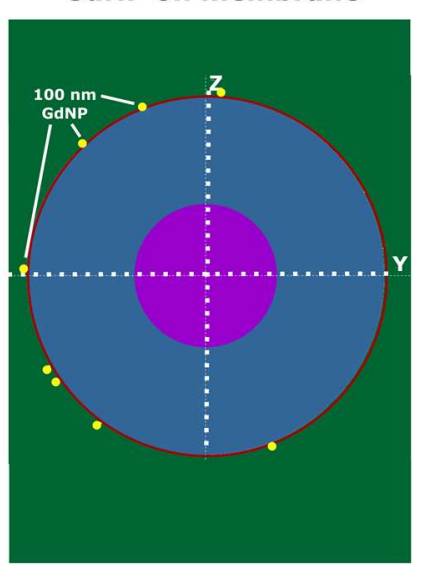

Figure 1: Geometries taken into account in the simulations for the different gadolinium distributions: A) homogeneous distribution of Gd-atoms in cytoplasm, B) accumulation in lysosomes, C) $50 \mathrm{~nm}$-nanoparticles randomly distributed in the cytoplasm and D) $50 \mathrm{~nm}$ nanoparticles randomly distributed on the membrane. The gadolinium mass was $0.6 \mathrm{pg} / \mathrm{cell}$ in all conditions.

The secondary electron spectra coming from Gd interactions were also computed. The electrons passing through the nucleus were analyzed and relative spectra were obtained as the ratio of 
252 electrons collected in the presence of gadolinium versus the electrons collected in the control 253 condition.

254 I.3. Monte Carlo Simulations around a single nanoparticle

255 In order to quantify the dose-enhancement due to low-energy secondary particles in the immediate 256 vicinity of the nanoparticles, a study was carried out at a smaller scale using a geometry approaching 257 those of previous works ${ }^{14,16,19}$. It consisted in the calculation of DEF in a $1 \mu \mathrm{m}$ diameter water sphere 258 in whose center a single GdNP is placed. Simulations were performed for GdNPs of different sizes 259 (radius from 1 to $50 \mathrm{~nm}$ ) and for different beam energies (from $25 \mathrm{keV}$ to $1.25 \mathrm{MeV}$ ). The source 260 was circular with the same radius as the nanoparticle and located just in front of it. Note that all the 261 photons emitted from the source intercept the nanoparticle. This geometry was chosen to keep a 262 reasonable computation time (about $12 \mathrm{~h}$ per beam energy and per GdNP size using 24 CPUs) with 263 acceptable statistical errors (<3\%).

264 II. Results

\section{II.1 Monte Carlo Simulations: Intracellular Gd Distribution}

266 The dose-enhancements calculated at the sub-cellular scale are shown in Figures 2 and 3 for the various gadolinium distributions described in section I.2. Figure 2 shows the dose-enhancement maps for the beam energies bracketing the gadolinium K-edge (50 and $52 \mathrm{keV}$ ), the energy for which the maximum radiosensitization effect ( $65 \mathrm{keV}$ ) was observed ${ }^{7}$, and the cobalt-source mean energy 270 (1250 keV). The color scale maximum (Figure 2) was set to DEF = 3 for better readability. However, 271 the dose-enhancements were much larger in the close vicinity of the NPs and the "lysosomes". The 272 maxima observed with the homogeneous Gd-atom distribution are much smaller than those 273 observed with nanoparticles. In all cases, the DEFs at high energy are close to 1 . For kilovolt energies, 274 Gd strongly enhances the doses and the DEF is found to be larger above the Gd K-edge than below 275 it (Figure 2). 
$27765 \mathrm{keV}$ beam energies respectively. This tremendous dose increase may be explained by the high 278 production of LEE $(<2 \mathrm{keV})$ following the photoelectric interactions that occur in the gadolinium 279 clusters. These are essentially $\mathrm{K}$ photoelectrons as well as Auger and Coster-Krönig electrons 280 resulting from atomic relaxation and secondary photoelectric interaction cascades.

\section{A)}

Homogeneous

B) $50 \mathrm{keV}$

Z

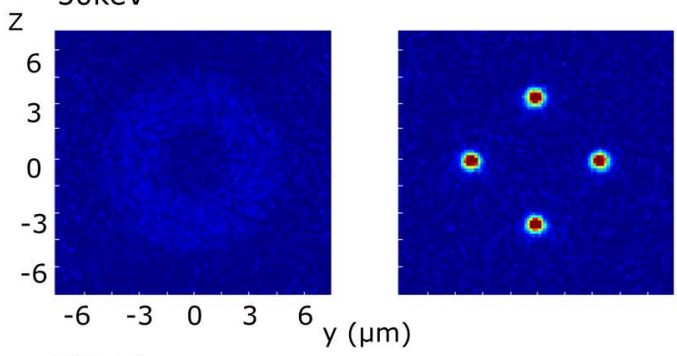

$52 \mathrm{keV}$

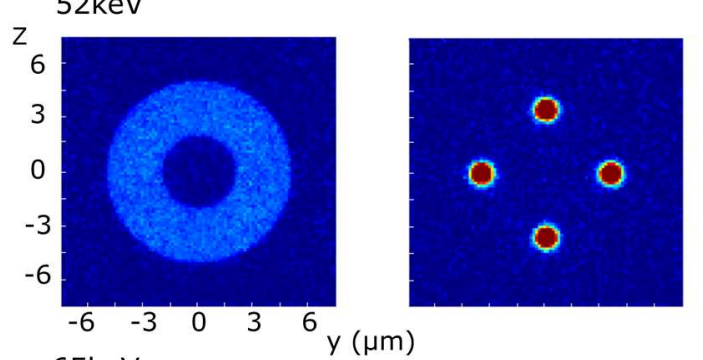
$65 \mathrm{keV}$

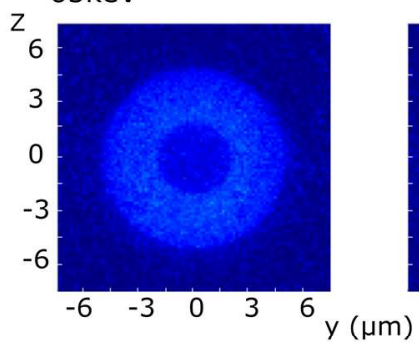

$1250 \mathrm{keV}$ (60Co)

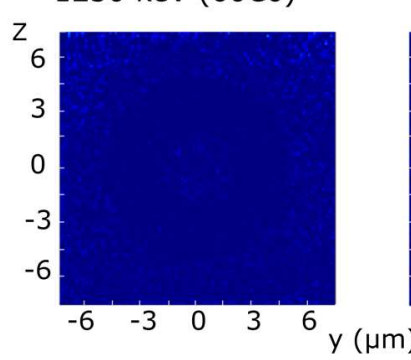

C)

\section{GdNP inside}

cytoplasm
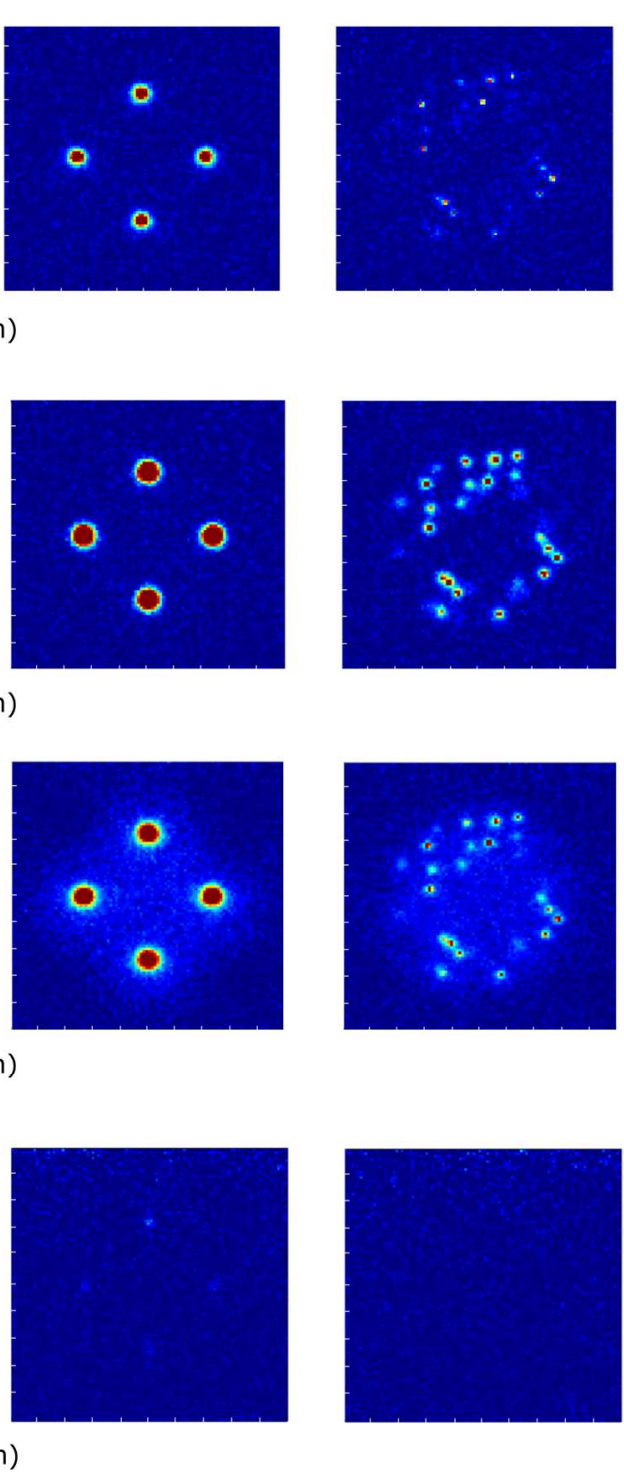

D)
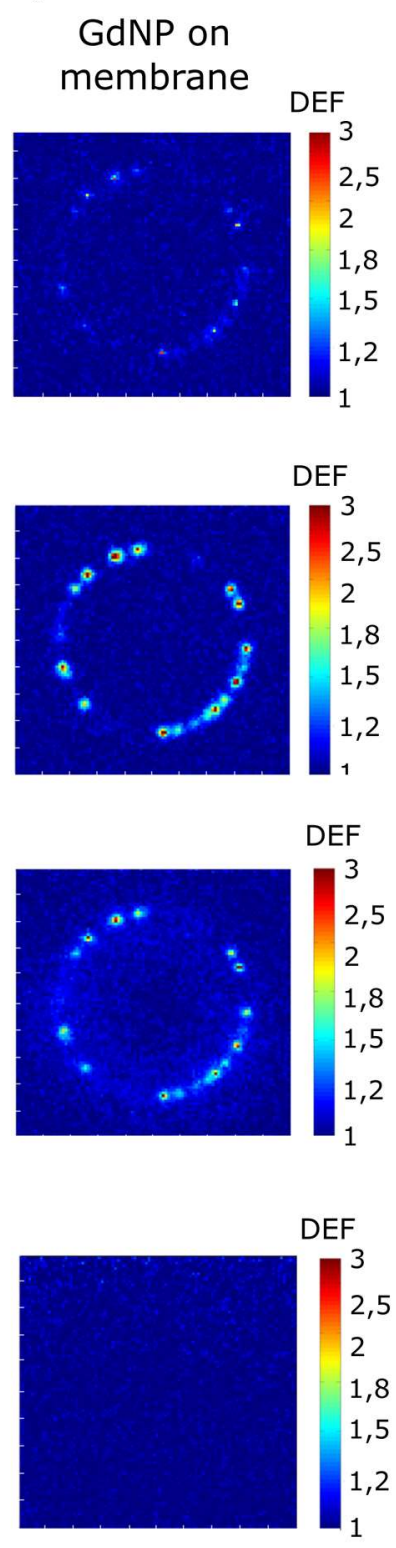

Figure 2: DEFs obtained at 50,52, 65 and $1250 \mathrm{keV}$ for the different gadolinium distributions: A) homogeneous Gd-atom distribution in the cytoplasm, B) accumulation of Gd-atoms in lysosomes, C) $50 \mathrm{~nm}$ radius nanoparticles distributed in cytoplasm and D) $50 \mathrm{~nm}$ radius nanoparticles distributed on the membrane. The gadolinium mass was $0.6 \mathrm{pg} / \mathrm{cell}$ in all conditions. $Y$ and $Z$ scales are in micrometers. The pixel resolution is $150 \mathrm{~nm}$. 
micro-distributions and for photon energies ranging from $25 \mathrm{keV}$ to $1250 \mathrm{keV}$.
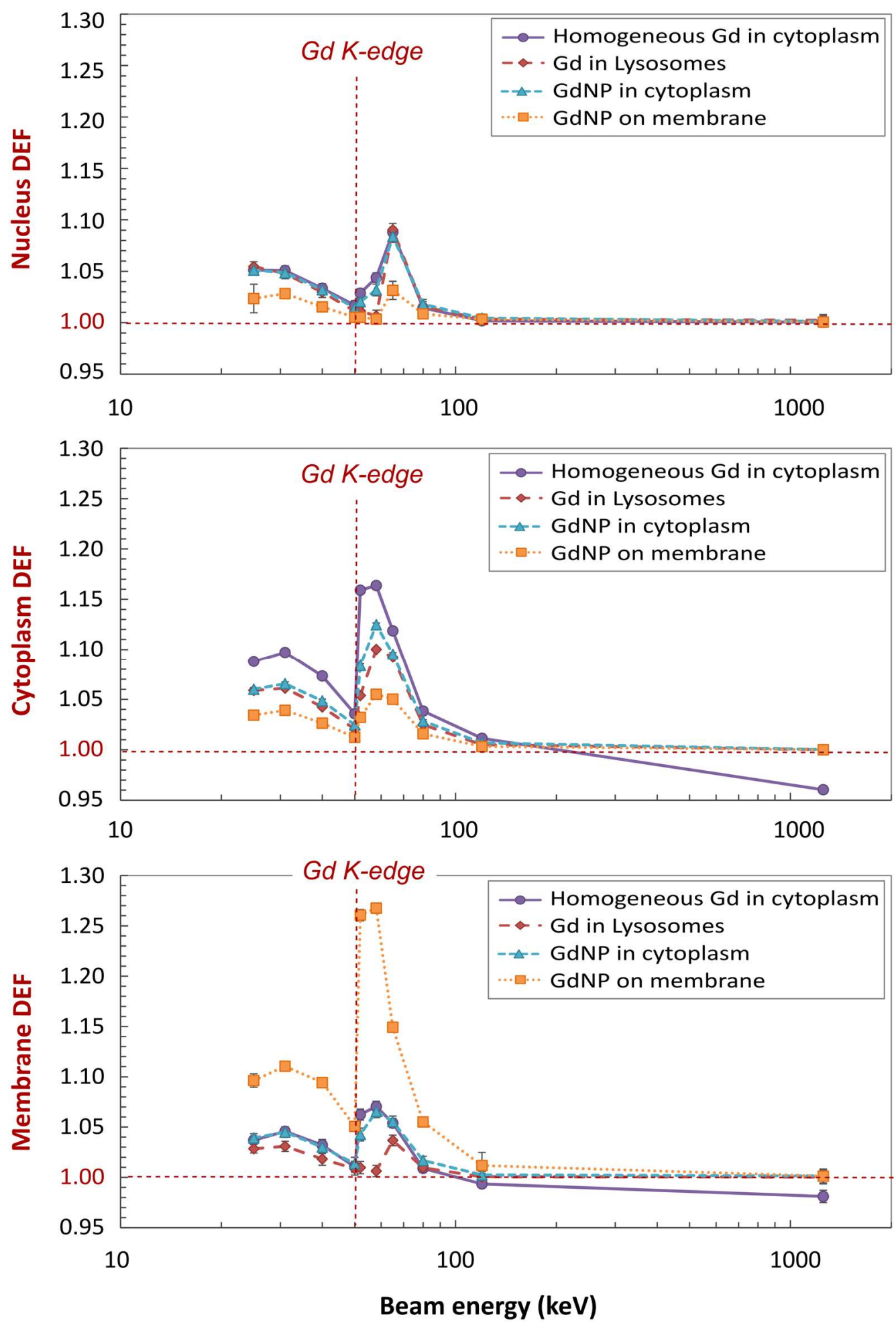

Figure 3: Mean DEFs calculated to the nucleus, cytoplasm and membrane as a function of the beam energy (from $25 \mathrm{keV}$ to $1250 \mathrm{keV}$ ) for four gadolinium distributions with $0.6 \mathrm{pg}$ Gd/cell: homogeneous Gd-atom distribution in cytoplasm (-), accumulation of Gd-atoms in "lysosomes" ( $\diamond), 50 \mathrm{~nm}$ radius GdNPs distributed in cytoplasm (\$) and $50 \mathrm{~nm}$ radius GdNPs distributed on the membrane ( $\square$ ). Uncertainties of confidence interval 3 sigma are close to $1 \%$. 
In all the cases, the nucleus exhibits the lowest mean $\operatorname{DEF}(<1.10)$ with a maximum value at

$29465 \mathrm{keV}$ regardless of the Gd distribution as long as $\mathrm{Gd}$ is in the cytoplasm. For the cytoplasm and the 295 membrane, the K-edge is much sharper with a maximum around $58 \mathrm{keV}$. The mean DEF values are larger, up to 1.16 for the cytoplasm in the case of homogeneous Gd-atoms (representing GdCA) and 1.27 for the membrane when the GdNPs are distributed around the membrane. It is worth mentioning that in all cases the DEF is very close to 1 for energies above $100 \mathrm{keV}$.

\section{II.2 Experimental results and comparison with the simulations}

The alpha and beta parameters calculated from the linear quadratic fits of the dose-survival plots without Gd are given Table 1. They were measured at $33 \mathrm{keV}, 50 \mathrm{keV}$ and $1.25 \mathrm{MeV}$.

\begin{tabular}{ccc}
\hline Beam Energy (keV) & $\alpha \pm \sigma_{\alpha}\left(\mathrm{Gy}^{-1}\right)$ & $\beta \pm \sigma_{\beta}\left(\mathrm{Gy}^{-2}\right)$ \\
\hline $33 \mathrm{keV}$ & $0.134(0.015)$ & $0.030(0.013)$ \\
$50 \mathrm{keV}$ & $0.014(0.013)$ & $0.045(0.018)$ \\
$1.25 \mathrm{MeV}$ & $0.221(0.020)$ & $0.009(0.003)$
\end{tabular}

Table 1: Results of the fit of the linear-quadratic model (Eq. 1): parameters $\alpha$ and $B$ and their standard deviation in parenthesis. The survival curves versus $x$-ray dose were measured for irradiations without $\mathrm{Gd}$ at $33 \mathrm{keV}, 50 \mathrm{keV}$ and $1.25 \mathrm{MeV}$, i.e. low, intermediate and high-energy range, respectively.

The Gd uptake of the F98 cells in presence of GdNPs was measured by means of ICP-MS. The Gd uptake in the rinsed cells, measured after $5 \mathrm{~h}$ incubation with GdNPs, was $0.6 \pm 0.05 \mathrm{pg}$ Gd/cell. The remaining $\mathrm{Gd}$ concentration in the culture medium at the end of the incubation period was $1.8 \pm 0.05 \mathrm{mg} \mathrm{Gd} / \mathrm{mL}$ (un-rinsed case). The kinetics for the uptake of GdNPs by F98 cells was reported in our previous publication ${ }^{7}$. No toxicity was observed when cells were incubated with GdNPs without irradiation. The experimental DEFs versus x-ray energy are shown in Figure 4-A, they were calculated using Eq. 5 and the corresponding $\alpha$ and $\beta$ parameters. Figure $4-A$ shows the mean computational DEFs calculated in the nucleus, cytoplasm and membrane in comparison with the experimental DEF (DEFexp) measured after 5h of GdNP incubation with F98 cells (rinsed before 


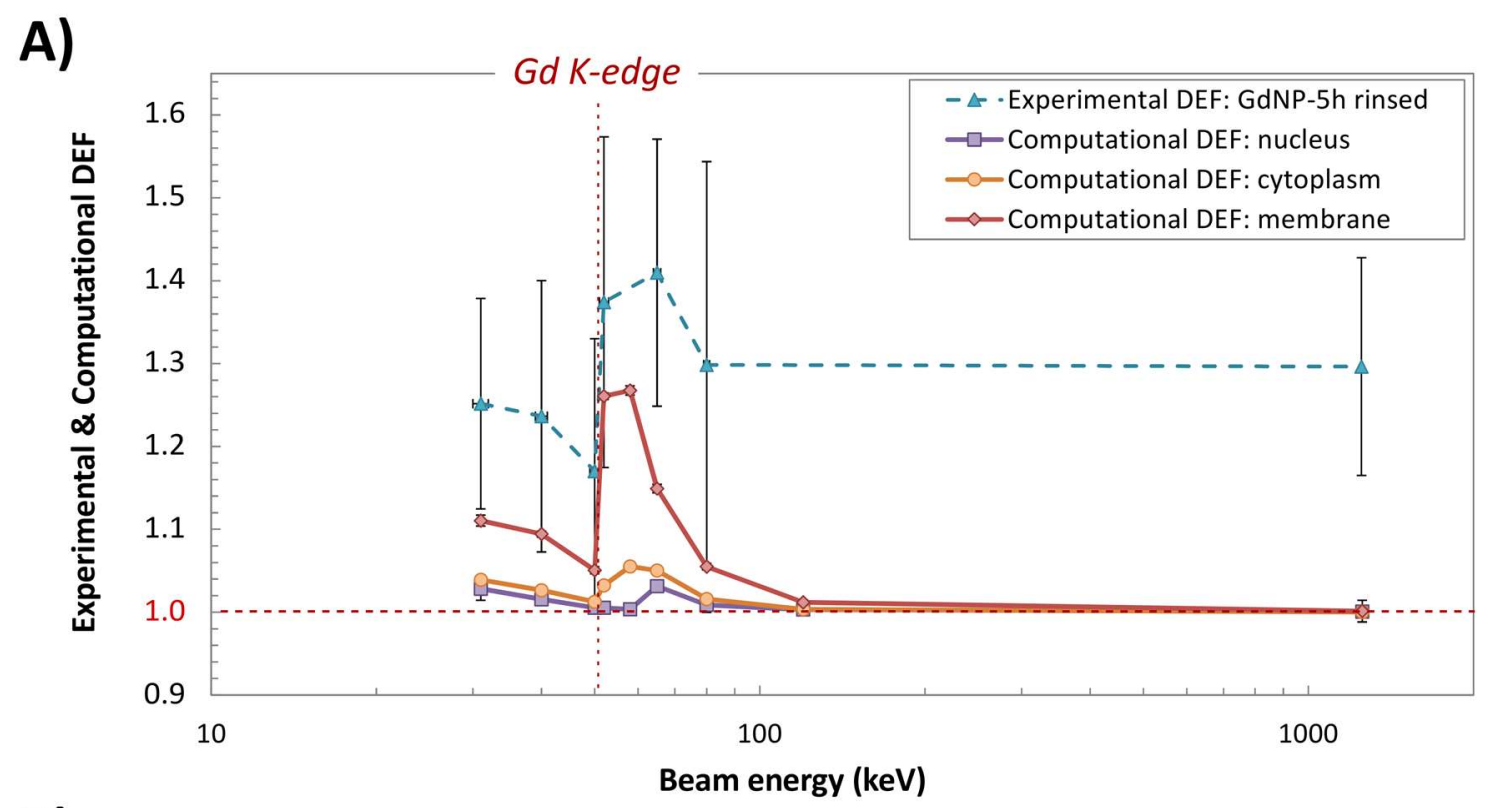

B)

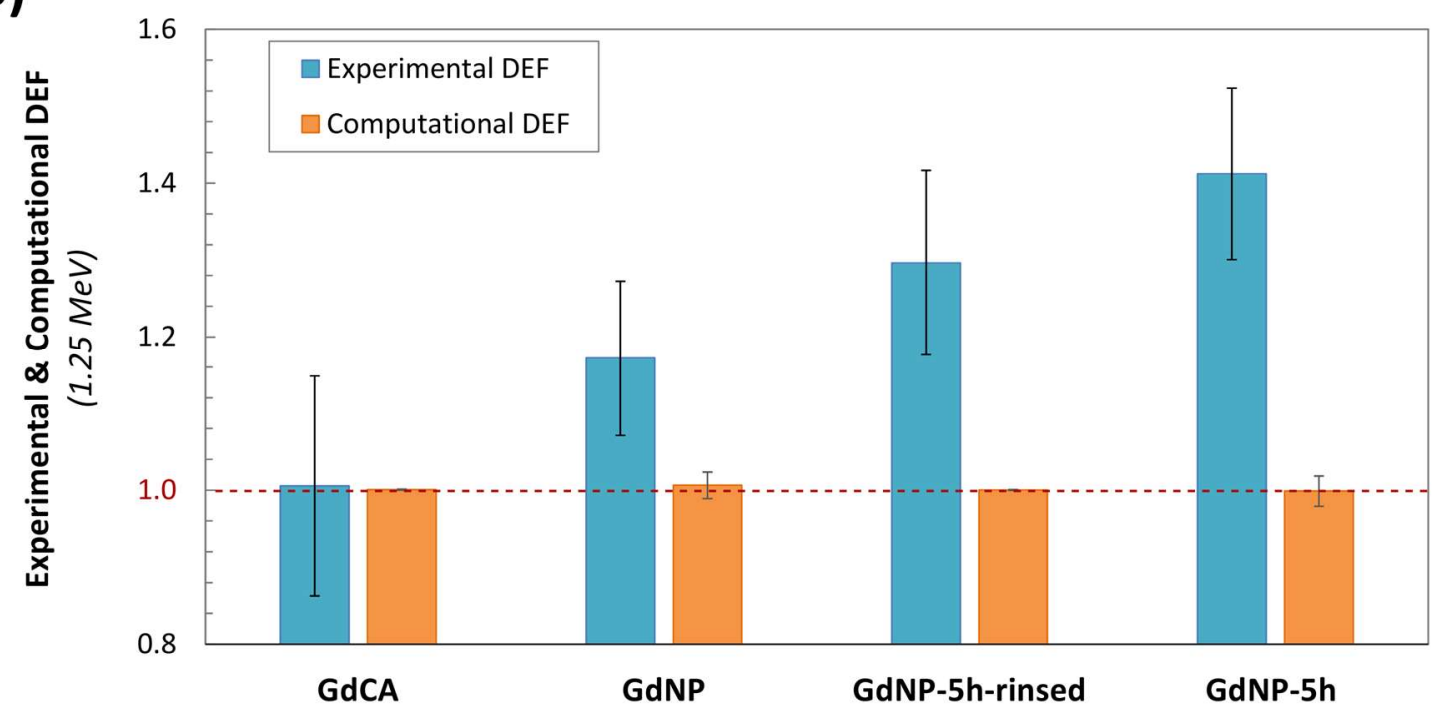

Figure 4: A) mean nucleus, cytoplasm and membrane DEF versus experimental $D E F$ ( $D E F_{\text {exp }}$ ) as a function of photon beam energy (from $31 \mathrm{keV}$ to $1.25 \mathrm{MeV}$ ). Numerical calculations correspond to $50 \mathrm{~nm}$ - GdNPs randomly distributed at the surface of the cell membrane. B) Computational DEF versus experimental DEF obtained with various Gd-treatment conditions: in presence of $2.1 \mathrm{mg} \mathrm{Gd} / \mathrm{mL} G d C A$ during irradiation (GdCA); in presence of $2.1 \mathrm{mg} \mathrm{Gd} / \mathrm{mL}$ GdNPs during irradiation (GdNP); 5 h incubation with $2.1 \mathrm{mg} \mathrm{Gd} / \mathrm{mL}$ GdNPs and cells rinsed before irradiation (GdNP-5h rinsed); $5 \mathrm{~h}$ incubation with $2.1 \mathrm{mg} \mathrm{Gd} / \mathrm{mL}$ GdNPs and cells not rinsed before irradiation (GdNP-5h). The mean relative errors on the survival data was about $10 \%$. Uncertainties of confidence interval $3 \sigma$ on computational DEF range from $1 \%$ to $3 \%$ (increasing with energy).

At all energies, experimental DEFs were found to be larger than the physical DEFs, but follow a 
329 In Figure $4-B$ is reported the comparison at high-energy of mean cell computational DEF versus 330 experimental DEF with various incubation conditions (no incubation and $5 \mathrm{~h}$ incubation, rinsed or 331 not before irradiation) and molecular shape (GdCA or GdNPs). An important radio-sensitivity is 332 observed at high energy (1.25 MeV) with GdNPs whereas no effect was observed in presence of 333 Magnevist $^{\circledR}$, as expected from modeling for all irradiation conditions. The maximum $\mathrm{DEF}_{\exp }(1.41 \pm$ 3340.11 ) is found for the cells incubated $5 \mathrm{~h}$ with GdNPs and irradiated with the remaining GdNPs in the 335 medium. The presence of GdNPs inside the cells (GdNP-5h rinsed) induces higher DEF $\exp (1.3 \pm 0.12)$ 336 observed when cells are irradiated in presence of GdNPs but without incubation (GdNP, $337 \mathrm{DEF}_{\exp }=1.17 \pm 0.1$ ), although the amount of $\mathrm{Gd}$ is far less in the first condition.

\section{II.3 Nanoparticle Size and Beam Energy at the Nanometer Level}

340 Figure 5 reports the computational DEF profiles calculated for a single GdNP. The aim was to 341 evaluate the relative influence of the GdNP size and the beam energy at the nanometer scale. The 342 first set of simulations was performed at $55 \mathrm{keV}$ with the GdNP radius varying from 1 to $50 \mathrm{~nm}$ (left 343 panel). The second set of simulations was performed with a $50 \mathrm{~nm}$ GdNP radius and with an X-ray 344 beam energy ranging from $25 \mathrm{keV}$ to $1.25 \mathrm{MeV}$ (cobalt source). 

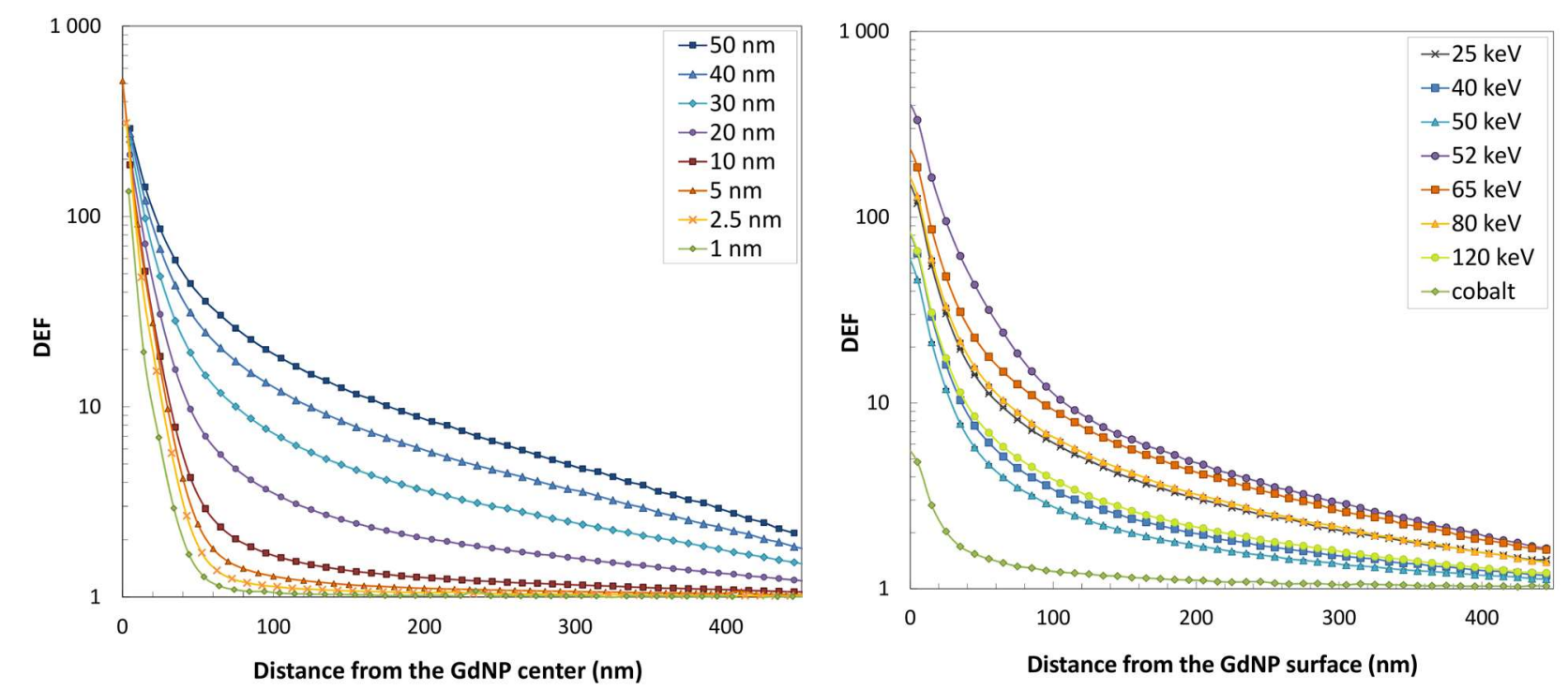

Figure 5: Left panel: DEFs calculated along the beam direction as a function of the distance from the GdNP surface, beam energy $55 \mathrm{keV}$ and GdNP radius from 1 to $50 \mathrm{~nm}$. Right panel: DEFs calculated along the beam direction as a function of the distance from the GdNP surface, $50 \mathrm{~nm}$ radius GdNP and beam energy from $25 \mathrm{keV}$ to $1250 \mathrm{keV}$ (cobalt source). Voxel size: $10 \mathrm{~nm}$.

All DEF profiles show a strong decrease with the distance from the NP surface. Their slopes greatly decrease over the first $100 \mathrm{~nm}$ (from the surface of the NP) and a softening slope beyond, with DEFs less than 10 for all energies. DEFs from 5 to 400 are obtained at the surface of the NP, with a minimum for the cobalt source energy and a maximum at $52 \mathrm{keV}$.

\section{II.4 Electron Spectra Reaching the Cell Nucleus}

Figure 6 - $A$ shows the probability density of electrons crossing the nucleus when the cell contains

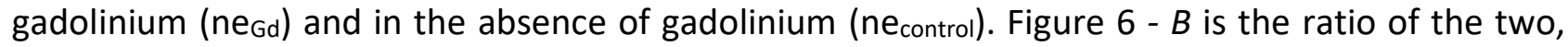


conditions). The beam energy was set to $58 \mathrm{keV}$, i.e. $7.8 \mathrm{keV}$ above the $\mathrm{Gd}$ K-edge.

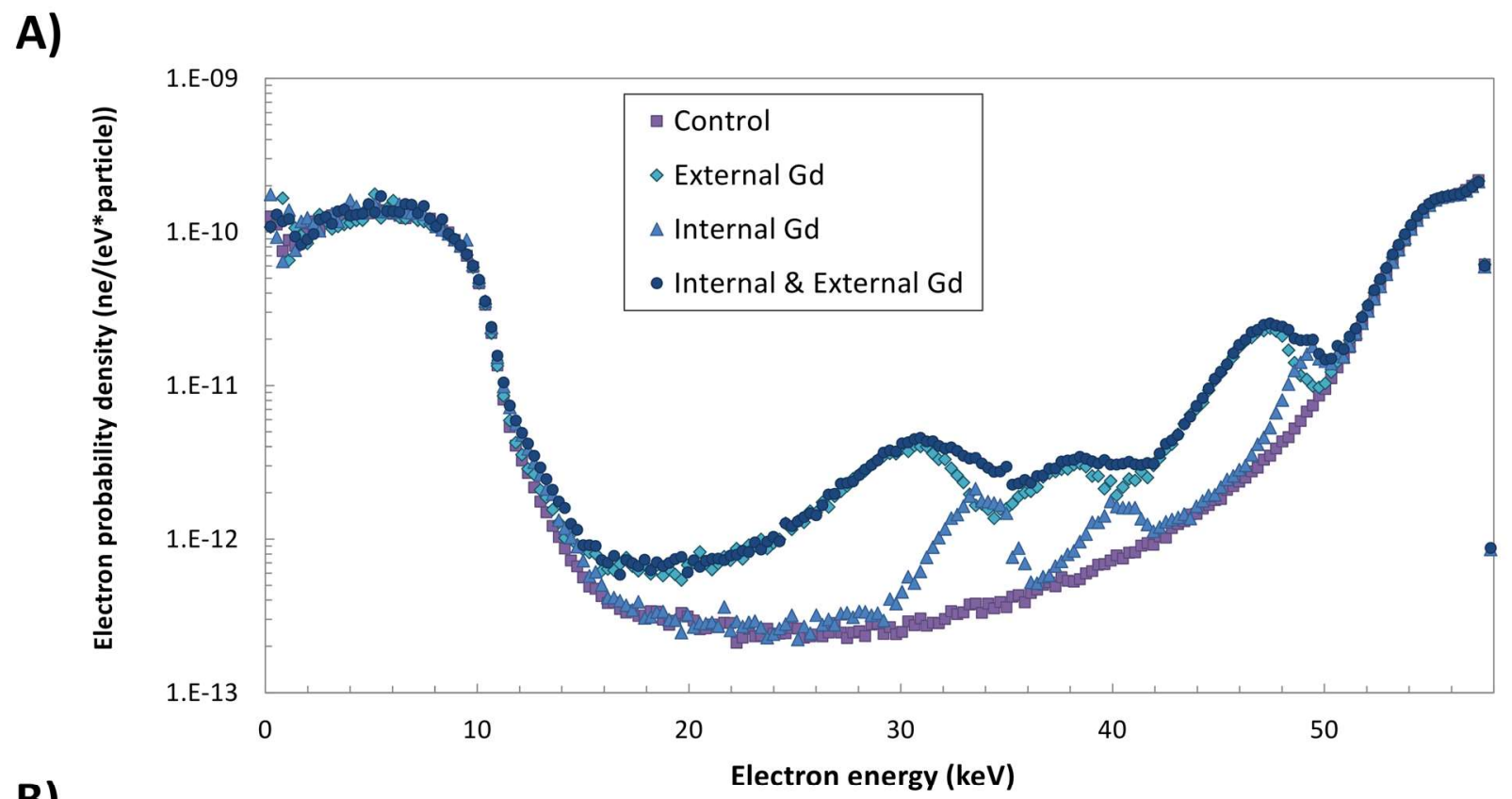

B)

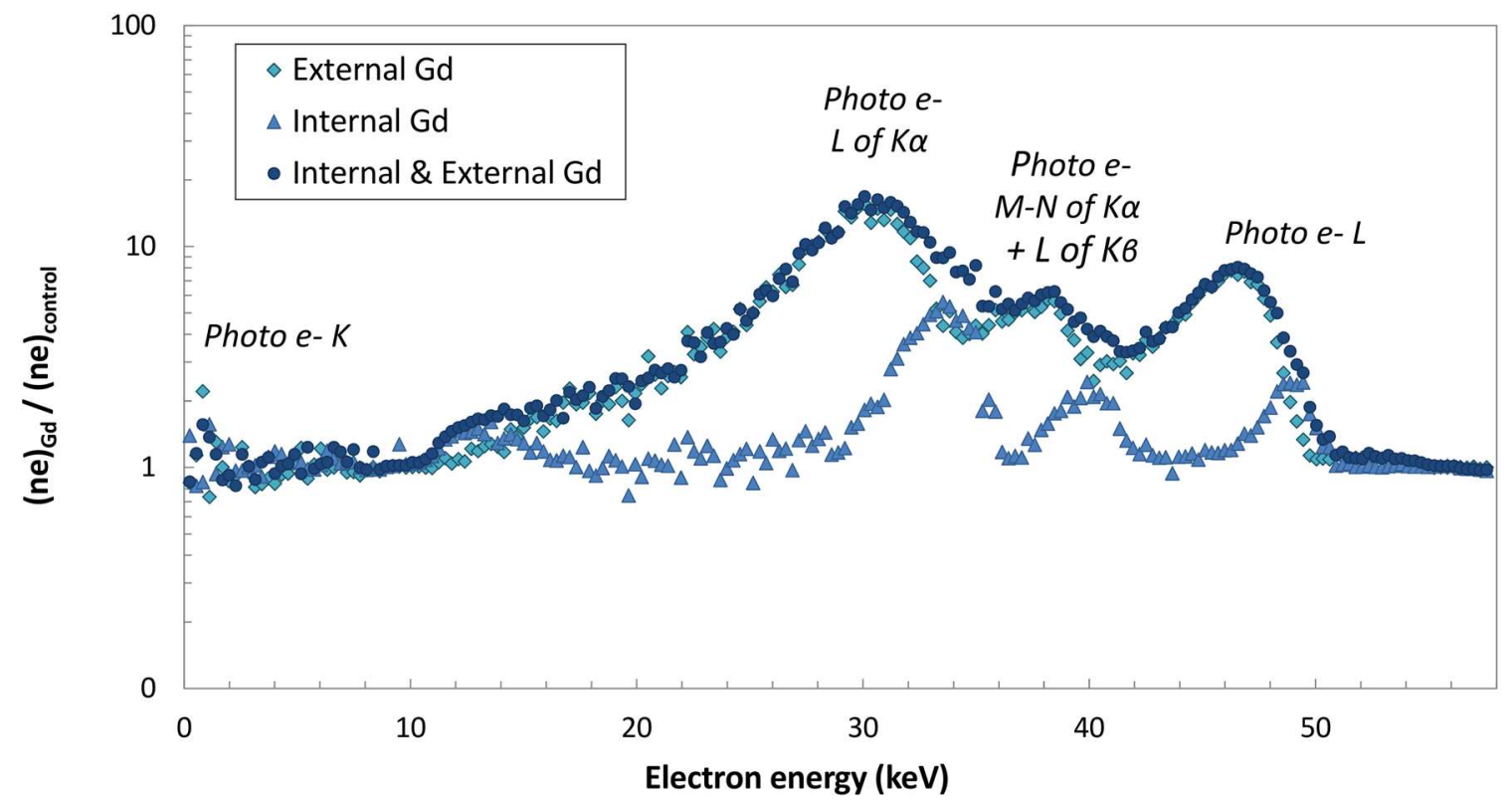

Figure 6: A: electron probability density versus the electron energy, where (ne) Gd refers to the number of electrons crossing the nucleus when $\mathrm{Gd}$ is distributed within extra-nucleus areas (cytoplasm ( $\mathbf{\Delta}$ ), external $(\diamond)$ and both $(\bullet))$ whereas (ne) control refers to the corresponding number in the absence of $\mathrm{Gd}(\mathrm{B})$. The X-ray beam energy is $58 \mathrm{keV}$. B: Ratio of the number of electrons crossing the nucleus in presence of Gd relative to the number of electrons crossing the nucleus without Gd: (ne) $)_{G d} /(n e)_{\text {control }}$ ratio.

371 The amplitudes of the characteristic peaks vary from 3 to 15, for electron energies between 20 and

$50 \mathrm{keV}$ (Figure 6B). This increase number of electrons reaching the nucleus due to Gd-interactions could be a cause of additional damages to the DNA, they are however not predominant in the electron spectra (Figure 6A). When the electrons are generated from Gd in the external medium, 
they have to travel through more material before reaching the nucleus, inducing a spread of the spectrum and wider energy bandwidths compared to the internal condition. The origin of the peaks was identified by correlating their position with the atomic relaxation tables and the gadolinium binding energies $(50.24 \mathrm{keV}, 7.24-8.38 \mathrm{keV}$ and $1.2-1.9 \mathrm{keV}$ for $\mathrm{K}, \mathrm{L} 1-\mathrm{L} 3$ and M1-M5 layers respectively). The peaks observed around 30 and $40 \mathrm{keV}$ are due to the electrons originated from the second photoelectric interactions of $K_{\alpha}$ and $K_{\beta}$ fluorescence photons. Those coming from the primary interactions on the $\mathrm{K}$ and $\mathrm{L}$ shells are found around 0.5 and $48 \mathrm{keV}$ respectively. The great majority of potential high-LET electrons ${ }^{22}$, K photoelectrons, Auger and Coster-Krönig electrons, are not able to reach the cell nucleus because of their very low energies $(<10 \mathrm{keV})$, even in the case of internal gadolinium.

\section{Discussion}

LEE and more particularly Auger electrons, were suggested in the literature as a key agent in the radiobiological effects of NPs, since they are able to induce lethal DNA breaks via direct or indirect ionization ${ }^{22}$. However, the NPs usually remain in the cytoplasm or on the cell membrane and do not reach the cell nucleus ${ }^{7,9,24,25}$. We have shown in this study that the LEE produced in the cytoplasm or in the extracellular area are mostly absorbed before reaching the cell nucleus, even with intracellular gadolinium (Figure 6). In addition, we have shown that the very strong dose gradients observed around the NPs extend only a few tens of nanometers from small NPs (Figure 5). Since the NPs are mostly located at a few micrometers from the nucleus, other components like the organelles in the cytoplasm or the cell membrane may be potential targets.

When the NPs are clustered on the cell membrane, the highest DEF (1.27) was found on the membrane with beam energy above the Gd K-edge (Figure $4-A$ ). The increase of dose can induce, by direct or indirect effects, a membrane degradation and lead to cell death ${ }^{36}$. Additional Gd distributions were simulated to model the case where the GdNPs accumulate in vesicles such as 
endosomes or lysosomes, such as reported by others ${ }^{25,37}$. Large clusters of gadolinium distributed into the cell cytoplasm were modeled and called "Iysosomes" configuration. Dose-enhancement factors up to 60 in the lysosomes were calculated (cf. Figure 2). These DEFs may also induce lethal damage to the cell since the endosomal and lysosomal systems have important functions for cell survival $^{9,37,38}$. Mitochondrial damage can also be a prevalent cause of cell death if NPs accumulate there ${ }^{10,18,24,26}$ and our geometry with GdNPs distributed in the cytoplasm (Figure 2) can represents the order of magnitude of achievable DEFs close to these structures. In good agreement with our results, Douglass et al., who modeled similar cellular geometry, suggested that LEE like Auger electrons coming from the NP have a negligible effect on the overall dose increase due to their small ranges ${ }^{15}$. The average dose-enhancement on the entire cell can be attributed mainly to higher energy photoelectrons. Note that our simulated cell model is spherical and implies a bias because of its symmetry and size. Indeed, cells actually have a more complex shape and the nucleus is sometimes very close to the cell membrane. The overall biological effect may be due to cumulative damage on the entire cell (nucleus, membrane and cytoplasm organelles). In a recent publication, Sung et al. $^{34}$ have evaluated the influence of the cell shape and nucleus location on doseenhancement produced by AuNPs ( $2 \% \mathrm{Au}$ in mass, distributed on the membrane of cells). They demonstrated that a small increase could be observed at $\mathrm{kV}$ energies (51 keV and $150 \mathrm{kVp}$ ) when the nucleus was shifted toward the membrane (up to 1.2 for F98 cell modelling), but no doseenhancement was obtained at high energies (6 MV). In addition, the shape of the cell did not significantly affect their results when the nucleus was placed in the cell center for the F98 cells.

Various characteristic variations of the DEFs with beam energy were observed for the different intracellular Gd distributions and the cell compartment considered. The results clearly demonstrate that the larger heterogeneities and clustering behavior in Gd distribution lead to larger local DEFs (Figure 2). This is due to a significant production of electrons by atomic relaxation cascades and to secondary photoelectric interactions of fluorescence photons, the latter being more probable for a 
higher density of Gd atoms. By contrast, homogeneously distributed Gd-atoms (representing GdCA) led to larger DEFs in the cytoplasm (Figure 3-Cytoplasm) in comparison with GdNPs. This is mainly due to the auto-absorption of LEE in the Gd-clustered structures. This suggests that if the GdCA could be internalized in the cytoplasm, the radiosensitizing effect on the cells could be potentially important. This warrants further experimental studies to compare internalized GdCA with GdNPs. In all conditions, the interactions beyond the Gd K-edge produce the higher DEFs (Figures 2 and 3). The DEF gradient as a function of the distance from Gd is very sharp at $52 \mathrm{keV}$ (Figures 2 and 5) because of the low-energy photoelectrons $(<2 \mathrm{keV})$. At $65 \mathrm{keV}$, maximum DEFs in the Gd clusters (lysosomes or GdNPs) are slightly lower than at $52 \mathrm{keV}$ but with a more spatially extended DEF above 1.5 (Figure 2). Such a DEF halo could reach various critical cellular targets and create potential cell death, as already mentioned.

The experimental DEFs (DEFexp) in presence of GdNPs incubated 5h with cells showed higher values than computational DEFs at all energies, with a maximum enhancement ratio at $65 \mathrm{keV}$ $\left(D_{E F}=1.41 \pm 0.16\right)$ and a sharp K-edge transition. As the membrane DEF attained the highest calculated value $(\mathrm{DEF}=1.27)$ and also showed a sharp K-edge transition with beam energy, membrane damage could partly explain the observed biological effect. Interestingly, the DEF $\mathrm{exp}_{\text {at }}$ 1.25 MeV (Figure $4-B$ ) obtained in presence of pre-incubated GdNPs (DEF $\exp =1.3 \pm 0.13$ ) was found to be much larger than the computational DEF obtained with the "GdNP on Membrane" condition $\left(D E F_{M C}=1.0 \pm 0.004\right)$. On the other hand, in presence of GdCA, no dose-enhancement was observed at high energy $\left(D F_{\exp }=1.01 \pm 0.14\right)$, as predicted by the simulations. The increased biological efficacy observed with GdNPs at high energy appears to be correlated with the degree of Gd agglomeration in the cells and the incubation time (Figure $4-B$ ). Indeed, the internalized GdNPs seem to have a much higher impact on the radiosensitivity than those remaining outside the cells, given the differences in $\mathrm{Gd}$ concentrations. The experimental sensitivity reported after incubation with GdNPs seemed to have two origins: one that is energy dependent and related to the physical 
dose-enhancement; and the second that is independent of the energy. In comparison with the condition of cells incubated during $5 \mathrm{~h}$ with GdNPs and irradiated without rinsing (reported in Taupin et $a .^{7}$ ), the experimental DEF with the rinsed cells (Figure 4-A) follows the same trend versus the energy.

At the nanoscale level, the DEF at significant distances from the NP surface (> $100 \mathrm{~nm}$ ) increases with the size of the NP (Figure 5). This is explained by an increase of gadolinium mass with NP size and consequently of the number of secondary LEE released. A hardening of the spectrum is observed (auto-absorption within the NP of the LEE generated deeply) inducing a spreading of the dose. One should note that these profiles have not been normalized by the NP mass in order to focus on the effect of an isolated Gd cluster. By doing so and additionally normalizing the photon flux passing through the GdNP (method used in the publication of Chow et al. ${ }^{39}$ ), the average DEFs in the water sphere were found to be larger for the smallest radii (data not shown). The NPs of small radius therefore seem the most "efficient" at the nanoscale level due to the larger amount of LEE that can be extracted from them. By extension, for an equal Gd concentration in the entire volume considered, isolated heavy atoms (as GdCA) should be the most efficient Gd form in terms of LEE production and local DEF. Our results confirm this idea on Figure 3 - Cytoplasm as slightly larger DEFs are obtained in the homogeneous conditions.

In terms of radiosensitization efficiency, if the cell survival is linked to the mean dose, it would be more appropriate to consider isolated atoms; on the other hand, if there is a specific biological target, large NPs close to it could be of particular interest to increase cell damage by inducing local hot spot of dose. However, to improve our understanding it would be crucial to consider the biological and chemical consequences of the presence of NP in a cell environment, using more specific radiobiological models (e.g. with Geant4-DNA and the LEM model).

It is noteworthy that monochromatic energies were used in the present study. The linear accelerators conventionally used in clinics produce broad photon spectra including a large 
contribution of photons around hundreds of keV. Polychromatic radiations might thus produce more significant radiation dose-enhancement effects than those obtained with cobalt 60 in the presence of NP, as discussed by McMahon et al. ${ }^{40,41}$. Anyhow, kilovolt energies remain the most adapted to this new therapy involving heavy atoms photo-activation since the dose-enhancements are always found to be much higher (factor $>10-100$ ) in this energy range. In clinical situations, a compromise must be found in terms of low energy between the attenuation of the primary beam in the patient and the number of interactions in the heavy material injected within the tumor. The optimal X-ray beam energy seems to be around $80 \mathrm{keV}$ according to the study by Edouard et al. ${ }^{11}$. In the perspective of patient's treatment, tumor accumulation of the GdCA and GdNPs should be taken into account and preclinical data indicated quite different in vivo results. One should note that the pharmacokinetics of GdCA is quite rapid, limiting their in vivo tumor uptake in quantity and residence-time. On the contrary, the GdNPs might present a more favorable pharmacokinetic leading to much higher tumor uptake and potentially to tumor cell internalization, and therefore a higher radiosensitization effect.

\section{Conclusions}

The parameters which influence the local dose-enhancement were studied with Monte Carlo simulations at a sub-cellular and nano-scale, in presence of GdNPs or GdCA and were compared with experimental in vitro measurements. A high heterogeneity of the Gd distribution, a massive production of low energy electrons around NPs and an optimal X-ray beam energy, above the Gd Kedge, were shown to be key factors to increase both microscopic doses and cells radio-sensitivity. The dose-enhancement calculated by Monte Carlo simulations at the sub-cellular scale account only for part of the biological response observed in presence of GdNPs. Higher experimental DEFs were observed at all energies, even at $1.25 \mathrm{MeV}$ although no dose-enhancement was predicted by the simulations. To understand these discrepancies, it would be worth refining the simulations by using 
more realistic geometries (such as the source and the cell model) and other codes adapted to nanodosimetry and chemistry modeling, such as Geant4-DNA. Performing further biological studies would also help to decipher the bio-mechanisms induced by the presence of GdNPs in the cells. On the other hand, the Monte Carlo simulations reflect the absence of dose-enhancement observed experimentally in presence of GdCA at high energy but suggest that high DEF would be obtained using GdCA in the energy range 50-65 keV. In conclusion, this study provides strong evidence that GdCA or GdNPs could both be used for radiation dose-enhancement therapy. Their biological distribution at the cellular scale will be the key factor for providing large dose-enhancements and this will determine their therapeutic efficacy.

\section{Acknowledgments:}

This work was performed within the ANR project 'Raphaelo' ANR-2010-BLAN-1532-02 and within the framework of the 'Labex Primes' (ANR-11-LABX-0063) operated by the French National Research Agency (ANR). Authors thank the laboratory of O. Tillement (Institut Lumière Matière, univ. Lyon, 69622 Villeurbanne cedex, France), for providing the nanoparticles that were used for experiments. We thank the European Synchrotron radiation Facility for providing beam time and T. Brochard for his technical assistance during the experiments. Authors are very grateful to E. Huffer and to L. Sancey, for their careful review and constructive advice.

\section{Conflict of interest:}

The authors have no relevant conflicts of interest to disclose.

\section{References:}

1. Brun E, Sanche L, Sicard-Roselli C. Parameters governing gold nanoparticle X-ray radiosensitization of DNA in solution. Colloids Surfaces B Biointerfaces. 2009;72(1):128-134.

2. Butterworth KT, McMahon SJ, Currell FJ, Prise KM. Physical Basis and Biological Mechanisms of Gold Nanoparticle Radiosensitization. Nanoscale. 2012. 
3. Hainfeld JF, Slatkin DN, Smilowitz HM. The use of gold nanoparticles to enhance radiotherapy in mice. Phys Med Biol. 2004;49(18):N309-N309.

4. Hainfeld JF, Smilowitz HM, O'Connor MJ, Dilmanian FA, Slatkin DN. Gold nanoparticle imaging and radiotherapy of brain tumors in mice. Nanomedicine. 2013;8(10):1601-1609.

5. Jain S, Coulter JA, Butterworth KT, et al. Gold nanoparticle cellular uptake, toxicity and radiosensitisation in hypoxic conditions. Radiother Oncol. 2014;110:342-347.

6. Sancey L, Lux F, Kotb S, et al. The use of theranostic gadolinium-based nanoprobes to improve radiotherapy efficacy. Br J Radiol. 2014;87(1041):20140134.

7. Taupin F, Flaender M, Delorme R, et al. Gadolinium nanoparticles and contrast agent as radiation sensitizers. Phys Med Biol. 2015;60:4449-4464.

8. Kotb S, Detappe A, Lux F, et al. Gadolinium-Based Nanoparticles and Radiation Therapy for Multiple Brain Melanoma Metastases: Proof of Concept before Phase I Trial. Theranostics. 2016;6(3):418-427. doi:10.7150/thno.14018.

9. Štefančíková L, Lacombe S, Salado D, et al. Effect of gadolinium-based nanoparticles on nuclear DNA damage and repair in glioblastoma tumor cells. J Nanobiotechnology. 2016;14(1):63. doi:10.1186/s12951-016-0215-8.

10. Taggart LE, McMahon SJ, Currell FJ, Prise KM, Butterworth KT. The role of mitochondrial function in gold nanoparticle mediated radiosensitisation. Cancer Nanotechnol. 2014;5(1):5. doi:10.1186/s12645-014-0005-7.

11. Edouard M, Broggio D, Prezado Y, Esteve F, Elleaume H, Adam JF. Treatment plans optimization for contrast-enhanced synchrotron stereotactic radiotherapy. Med Phys. 2010;37(6):2445-2456.

12. Jones BL, Krishnan S, Cho SH. Estimation of microscopic dose enhancement factor around gold nanoparticles by Monte Carlo calculations. Med Phys. 2010;37(7):3809-3816.

13. Carter JD, Cheng NN, Qu Y, Suarez GD, Guo T. Nanoscale energy deposition by X-ray absorbing nanostructures. $J$ Phys Chem B. 2007;111(40):11622-11625. http://www.ncbi.nlm.nih.gov/pubmed/17854220.

14. Leung MKK, Chow JCL, Chithrani BD, Lee MJG, Oms B, Jaffray D a. Irradiation of gold nanoparticles by X-rays: Monte Carlo simulation of dose enhancements and the spatial properties of the secondary electrons production. Med Phys. 2011;38(2):624-631. doi:10.1118/1.3539623.

15. Douglass M, Bezak E, Penfold S. Monte Carlo investigation of the increased radiation deposition due to gold nanoparticles using kilovoltage and megavoltage photons in a $3 \mathrm{D}$ randomized cell model. Med Phys. 2013;40(7):71710.

16. Lechtman E, Chattopadhyay N, Cai Z, Mashouf S, Reilly R, Pignol JP. Implications on clinical scenario of gold nanoparticle radiosensitization in regards to photon energy, nanoparticle size, concentration and location. Phys Med Biol. 2011;56(15):4631.

17. Lechtman E, Mashouf S, Chattopadhyay N, et al. A Monte Carlo-based model of gold nanoparticle radiosensitization accounting for increased radiobiological effectiveness. Phys Med Biol. 2013;58(10):3075.

18. McMahon SJ, McNamara AL, Schuemann J, Prise KM, Paganetti H. Mitochondria as a target for radiosensitisation by gold nanoparticles. J Phys Conf Ser. 2017;777(1):12008. doi:10.1088/1742-6596/777/1/012008.

19. McMahon SJ, Hyland WB, Muir MF, et al. Biological consequences of nanoscale energy deposition near irradiated heavy atom nanoparticles. Sci Rep. 2011;1:1-9. doi:10.1038/srep00018.

20. Tsiamas P, Liu B, Cifter F, et al. Impact of beam quality on megavoltage radiotherapy treatment techniques utilizing gold nanoparticles for dose enhancement. Phys Med Biol. 2013;58(3):451.

21. Lin Y, McMahon SJ, Paganetti H, Schuemann J. Biological modeling of gold nanoparticle enhanced radiotherapy for proton therapy. Phys Med Biol. 2015;60(10):4149.

22. Nikjoo H, Lindborg L. RBE of low energy electrons and photons. Phys Med Biol. 
2010;55(10):R65-R109. doi:10.1088/0031-9155/55/10/R01.

23. McMahon SJ, Hyland WB, Muir MF, et al. Nanodosimetric effects of gold nanoparticles in megavoltage radiation therapy. Radiother Oncol. 2011;100(3):412-416. doi:10.1016/j.radonc.2011.08.026.

24. Miladi I, Aloy M-T, Armandy E, et al. Combining ultrasmall gadolinium-based nanoparticles with photon irradiation overcomes radioresistance of head and neck squamous cell carcinoma. Nanomedicine Nanotechnology, Biol Med. 2015;11(1):247-257.

25. Rima W, Sancey L, Aloy M-T, et al. Internalization pathways into cancer cells of gadoliniumbased radiosensitizing nanoparticles. Biomaterials. 2013;34(1):181-195.

26. McNamara AL, Kam WWY, Scales N, et al. Dose enhancement effects to the nucleus and mitochondria from gold nanoparticles in the cytosol. Phys Med Biol. 2016;61(16):5993-6010. doi:10.1088/0031-9155/61/16/5993.

27. Porcel E, Tillement O, Lux F, et al. Gadolinium-based nanoparticles to improve the hadrontherapy performances. Nanomedicine Nanotechnology, Biol Med. 2014;10(8):16011608.

28. Miladi I, Le Duc G, Kryza D, et al. Biodistribution of ultra small gadolinium-based nanoparticles as theranostic agent: Application to brain tumors. $J$ Biomater Appl. 2013;28(3):385-394.

29. Verry C, Dufort S, Barbier EL, et al. MRI-guided clinical 6-MV radiosensitization of glioma using a unique gadolinium-based nanoparticles injection. Nanomedicine. 2016;11(18):24052417. doi:10.2217/nnm-2016-0203.

30. Di Corato R, Gazeau F, Le Visage C, et al. High-resolution cellular MRI: gadolinium and iron oxide nanoparticles for in-depth dual-cell imaging of engineered tissue constructs. ACS Nano. 2013;7(9):7500-7512.

31. Stasio G De, Rajesh D, Casalbore P, et al. Are gadolinium contrast agents suitable for gadolinium neutron capture therapy? 2005;27:387-398.

32. Salvat F. The penelope code system. Specific features and recent improvements. Ann Nucl Energy. 2015;82:98-109. doi:10.1016/j.anucene.2014.08.007.

33. Salvat F, Fernández-Varea JM, Sempau J. PENELOPE-2008. In: NEA Workshop Proceedings, Barcelona, Spain .; 2008.

34. Sung W, Ye S-J, Mcnamara AL, et al. Dependence of gold nanoparticle radiosensitization on cell geometry. 2017. doi:10.1039/c7nr01024a.

35. Bobyk L, Edouard M, Deman P, et al. Photoactivation of gold nanoparticles for glioma treatment. Nanomedicine Nanotechnology, Biol Med. 2013;9(7):1089-1097.

36. Buja LM, Eigenbrodt ML, Eigenbrodt EH. Apoptosis and necrosis. Basic types and mechanisms of cell death. Arch Pathol \&amp; Lab Med. 1993;117(12):1208-1214.

37. Stefancikova L, Porcel E, Eustache P, et al. Cell localisation of gadolinium-based nanoparticles and related radiosensitising efficacy in glioblastoma cells. Cancer Nanotechnol. 2014;5(1):115.

38. Turk B, Turk V. Lysosomes as "Suicide Bags" in Cell Death: Myth or Reality? J Biol Chem. 2009;284(33):21783-21787.

39. Chow JCL, Leung MKK, Jaffray D a. Monte Carlo simulation on a gold nanoparticle irradiated by electron beams. Phys Med Biol. 2012;57(11):3323-3331. doi:10.1088/00319155/57/11/3323.

40. Jain S, Coulter JA, Hounsell AR, et al. Cell-specific radiosensitization by gold nanoparticles at megavoltage radiation energies. Int J Radiat Oncol Biol Phys. 2011;79(2):531-539.

41. McMahon SJ, Hyland WB, Muir MF, et al. Nanodosimetric effects of gold nanoparticles in megavoltage radiation therapy. Radiother Oncol. 2011;100(3):412-416. 